\title{
An Artemisia WD40-Repeat Gene Regulates Multiple Cellular Functions in Arabidopsis
}

\author{
Wei Wang1,2, Qing Zhang1, Dianjing Guo ${ }^{1 *}$ \\ ${ }^{1}$ State Key Laboratory of Agrobiotechnology and School of Life Sciences, The Chinese University of Hong Kong, \\ Hong Kong, China \\ ${ }^{2}$ Current Address: Department of Biology, New York University, New York, USA \\ Email: *djguo@cuhk.edu.hk
}

Received 10 March 2016; accepted 9 May 2016; published 12 May 2016

Copyright @ 2016 by authors and Scientific Research Publishing Inc.

This work is licensed under the Creative Commons Attribution International License (CC BY). http://creativecommons.org/licenses/by/4.0/

(c) (i) Open Access

\begin{abstract}
In this study, we isolated a WD40-repeat gene from Artemisia annua glandular trichomes. This gene shows $69.97 \%$ sequence similarity to Arabidopsis TTG1 at aminoacid level. Sub-cellular localization study shows that AaWD40 protein diffuses in both cell nucleus and cytosol. The correct nuclear localization of AaWD40 was observed when co-expressed with AabHLH, a putative A. thaliana AtTTG1 homologue cloned from Artemisia annua glandular trichomes. When AaWD40 gene was ectopically over expressed in Arabidopsis transparent testa glabrous1-1 (ttg1-1) mutants of $A$. thaliana, PAs production in seeds was restored, and the trichomeless phenotypes of mutant were rescued. Real-time PCR analysis results revealed that ETC1, CPC, TTG2 and BAN (the downstream targets of AtTTG1 depend on regulatory complex), which regulate the epidermal differentiation and anthocyanin biosynthesis were differentially expressed as a result of AaWD40 over expression. Furthermore, the CLV1, CLV2, CLV3 and WUS, which are required to maintain the stem-cell niche of Arabidopsis shoot apex, were also modulated by AaWD40 and Arabidopsis TTG1. The transcriptions of AP2/ERF, bHLH, MYB, WRKY and NACs family proteins, which are mostly involved in defense, stress response and development regulation, were remarkably modulated by AaWD40 over expression. We hypothesize that WD40 repeat proteins act as a crucial factor in regulating a wide variety of cellular functions in $A$. thaliana.
\end{abstract}

\section{Keywords}

WD40 Repeat Protein, Glandular Trichome, Arabidopsis

"Corresponding author. 


\section{Introduction}

TWD repeat (WDR) proteins are characterized by 4 - 16 tandem WD (also called Trp-Asp or WD-40) motifs, which comprise 44 - 60 residue sequence typically delineated by the Gly-His (GH) dipeptide 11 - 24 residues from its N-terminus and ending in Trp-Asp (WD) dipeptide. In between GH and WD, a core sequence locates, which exhibits only limited sequence conservation [1]. The WD-repeat proteins are found in alleukaryotes but not in prokaryotes. They are implicated with a wide range of diverse functions, such as, cell division, cell-fate determination, signal transduction, cytoskeleton dynamics, protein trafficking, nuclear export, RNA processing and chromatin modification [2]. Currently, it is believed that WDR proteins provide binding sites and foster transient interactions for other proteins. In some cases, WDR proteins act as integral component of protein complexes or modular interaction domain of larger proteins by bringing the protein and associated ancillary domain(s) into proximity of its target(s) [2]. One of the most well studied WDR proteins is TRANSPARENT TESTA GLABRA1 (AtTTG1), which regulates several developmental and biochemical pathways, including the formation of root hair, differentiation of trichome and the production of seed mucilage and anthocyanin pigments in Arabidopsis [3]-[5]. A glabrous phenotype on leaf surfaces or stembase is observed in Arabidopsis ttg1 mutants. Other phenotypes include: absence of purple anthocyanin pigments in all tissues, absence of ruthenium red-staining seed mucilage, yellow seeds due to the absence of brown pigment in seed coat, and abnormal cell appearance of dry seed coat under scanning electron microscope [6]. It is believed that Arabidopsis TTG1 and the R2R3-MYB transcription factor GLABRA1 (GL1) [7] bind simultaneously to the basic helix-loop-helix (bHLH) proteins GLABRA3 (GL3) and ENHANCER OFGLABRA3 (EGL3) [8] to form a trimeric regulatory complex, thereby activating the transcription of downstream genes controlling epidermal differentiation and anthocyanin biosynthesis. More recent study found that ttg1-1 mutants tend to have more inflorescences compared to the wild type [9], indicating that TTG1 may also regulate genes involved in shoot development. Although one possible explanation is that the perturbation of flavonoids may directly affect auxin movement and consequently cause the developmental abnormality [10], it is still unclear whether flavonoids act directly as regulatory agents or indirectly through auxin accumulation or movement, or alternatively, TTG1 may regulate the shoot development directly.

In this work, we focus on functional study of an A. annua WD40 repeat gene, AaWD40, using cell biology, molecular biology, and functional genomics approach. We found that over-expression of AaWD40 can fully rescue the trichomeless and PA phenotype in Arabidopsis ttg1-1 mutant. Subcellular localization study suggested that the translocation of AaWD40 into nuclear requires the assistance of bHLH family protein. Ectopic over expression of AaWD40 in Arabidopsis altered the expression of CLV1, CLV2, CLV3 and WUS transcripts, which are required to maintain the stem-cell niche of Arabidopsis shoot apex. Finally, microarray analysis revealed the possible new function of AaWD40 and Arabidopsis TTG1 in regulating plant development and response to biotic and abiotic stress.

\section{Results}

\subsection{Molecular Characterization of AaWD40}

Gene tag (Contig24501) encoding a putative WD40 protein was identified from an in-house-generated A. annua glandular trichome EST library. Tissue specific expression analysis showed that this contig was preferentially expressed in filamentous trichomess and roots, also in glandular trichomes and leaves, although with lower abundance [11]. A full length CDS of 999bp was obtained by RACE PCR, and hereafter was referred to as AaWD40. The deduced amino acid sequence has 60\% - 73\% similarity to other known WD40 repeat proteins from different plant species. Phylogenetic analysis showed that MtWD40-1 is most closely related to GhAtTTG1 from Gossypium hirsutum. The 2974 bp genomic sequence of AaWD40 spans 3 exons and 2 introns.

\subsection{Subcelluar Localization of AaWD40}

Unlike most nuclear-bound WD40 proteins, no typical nuclear localization signal (NLS) was detected in the N-terminal region of AaWD40 using PSORT. In root tip cells of transgenic Arabidopsis plants over expressing 35S::AaWD40::GFP, strong fluorescent signals were detected in both nuclear and cytoplasmic were detected in both nuclear and cytoplasmic membrane (Figure 1). A similar expression pattern was also observed for AtTTG1. When 35S::AtTTG1::GFP was expressed in transgenic Arabidopsis plant or protoplasts, strong fluorescence sig- 


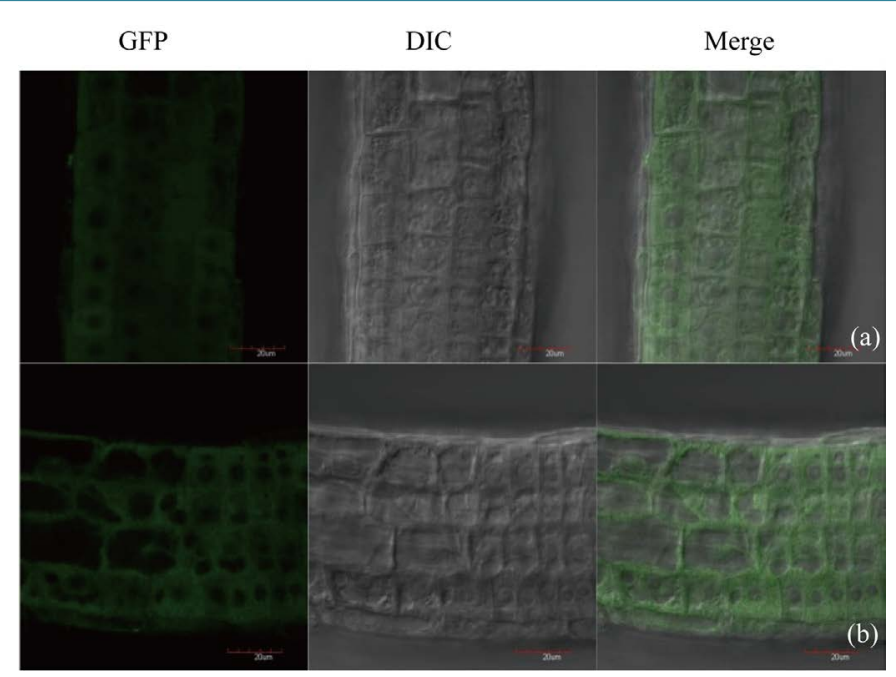

Figure 1. Subcellular localization analysis of AaWD40 and AtTTG1 in Arabidopsis root tips. (a) 35S::AaWD40::GFP; (b) 35S::AtTTG1:: GFP. 35S::GFP was used as control.

nal was detected in both cytoplasm and nuclear. This result is somewhat contradictory to previous report that AtTTG1 was mostly located in the nuclear region [10]. Other WD40 repeat proteins from petunia (AN11) and Perilla (PFWD) have been suggested to have a cytoplasmic pattern [12]. To further investigate the subcellular translocation pattern of WD40 protein, AtTTG1::GFP and AaWD40::GFP fusion proteins were co-expressed with AabHLH::RFP fusion protein in Arabidopsis protoplast respectively. As shown in Figure 2, coexpression of these two proteins resulted in an obvious nuclear pattern of AtTTG1 and AaWD40. These results indicate that the protein-protein interactions betweenWD40 repeat protein and bHLH protein may facilitate the TF translocation into nucleus.

\subsection{Complementation of A. thaliana $\operatorname{ttg} 1-1$ Mutant by AaWD40}

Because AaWD40 shares high sequence similarity with Arabidopsis TTG1, we first examined if they are functional homologs using complementation test. The mutation of TTG1 results in severe defects in anthocyanin pigmentation, seed coat pigmentation, seed coat mucilage, root hair positioning, and trichome differentiation. When AaWD40 (35S::AaWD40::GFP fusion) was transformed into Arabidopsis ttg1-1 mutant, PA production in seeds was fully restored, and the trichomeless phenotypes was rescued (Figure 3).

\subsection{Effects of AaWD40 Over-Expression on Trichomes Morphogenesis and PA Biosynthesis}

In Arabidopsis, TTG1, GL1 and GL3 forms a trimericregulatory complex to activate the expression ofGLABRA2 (GL2), a homeo-domain Zip (HD-Zip) transcription factor, and TRANSPARENT TESTAGLABRA2 (TTG2), a WRKY factor [7]. MYB/bHLH/TTG1 regulatory complex also activates the expression of a subset of partially redundant single-repeat R3 MYB proteins, including CAPRICE (CPC), ENHANCEROF TRY and CPC1 (ETC1). CPC andETC1 play central roles in lateral inhibition, by inactivating specific components of the trimericregulatory complex. To characterize the effects ofAaWD40 on trichome morphogenesis, the mRNA transcripts of 4 target genes of MYB/bHLH/TTG1complex, including GL2, CPC, ETC, and TTG2, were evaluated by qRT-PCR (Figure 4). Arabidopsis ttg1-1 mutant and over-expressor line were used as reference for comparison. The mRNA level of GL2, which encodes a direct positive regulator of trichome morphogenesis located downstream of the trimeric complex, was significantly increased. The mRNAs of CPC, ETC, TTG2 were strongly down-regulated in Arabidopsis ttg1-1 mutant. In TTG1 and AaWD40 over-expression lines, the expression of TTG2 and was significantly up-regulated. This is different from that of CPC and ETC1. BANYULS (BAN), which encodes a core enzyme in PA biosynthesis, is another direct target of MYB/bHLH/AtTTG1 complex [13]. The mRNA transcripts of BAN were found to be decreased in the ttg1-1 mutants and increased in AtTTG1and AaWD40 over-expression lines (Figure 4). 


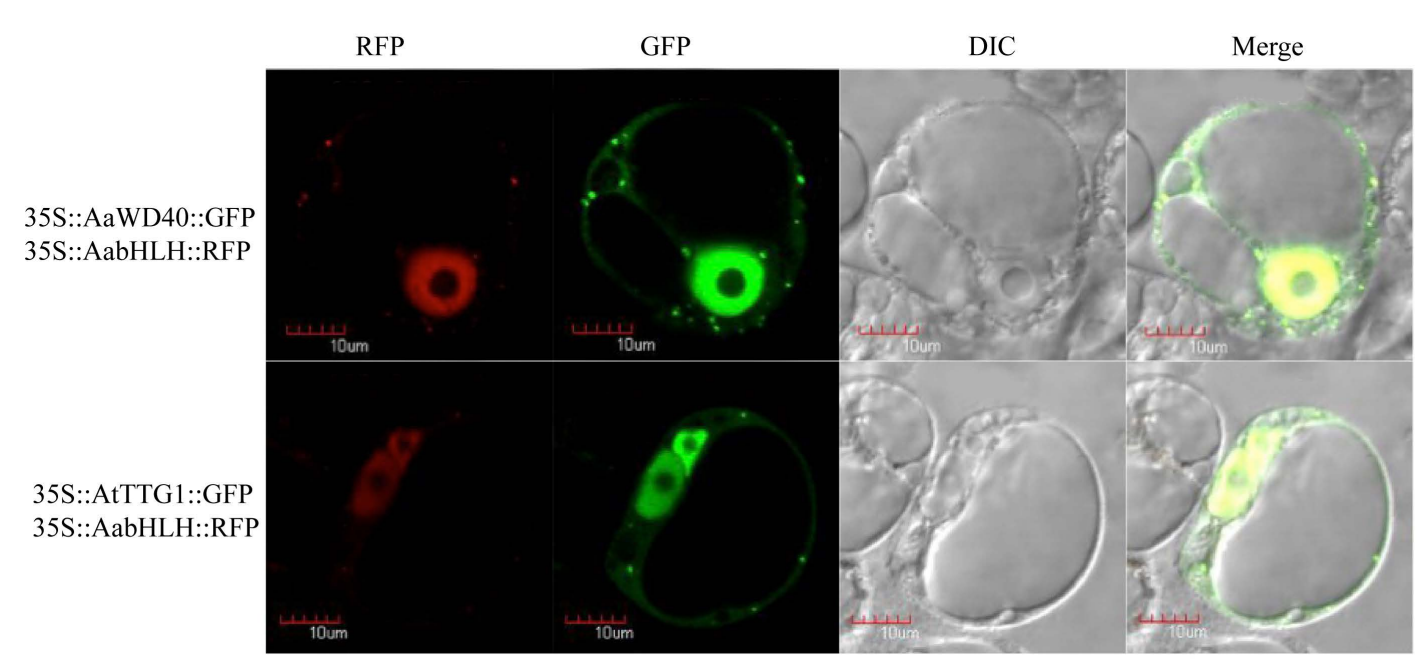

Figure 2. Co-expression of AaWD40 and AtTTG1 with AabHLH in protoplast. When a nuclear localized Artemisia bHLH protein (AabHLH) was coexpressing with either AaWD40 or TTG1, the nuclear signal was observed. Upper panel: Co-expression of 35S::AaWD40::GFP and 35S::AabHLH::RFP; Lower panel: Co-expression of 35S::AtTTG1::GFP and 35S::AabHLH::RFP.

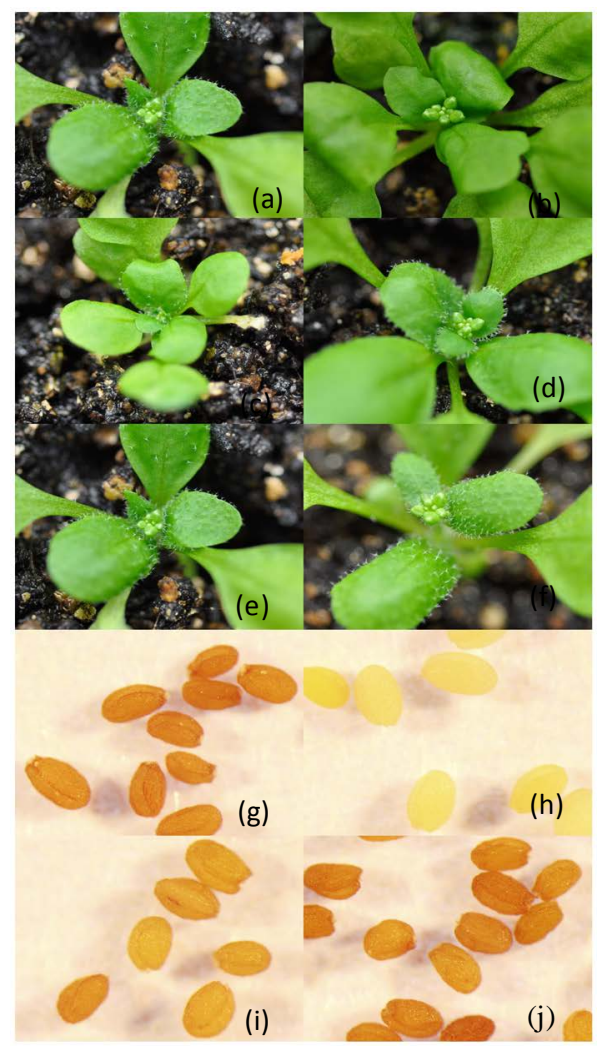

Figure 3. Genetic complementation of the Arabidopsis ttg1-1 mutant. (a) Leaves of the wild type (Ler); (b) Leaves of the ttg1-1 mutant line; (c) Leaves of the ttg1-1 mutant expressing 35S::ArtWD40::GFP (heterozygous); (d) Leaves of the ttg1-1 mutant expressing 35S::TTG1::GFP (heterozygous); (e) Leaves of the ttg1-1 mutant expressing 35S::ArtWD40::GFP (homozygous); (f) Leaves of the ttg1-1 mutant expressing 35S::TTG1::GFP (homozygous); (g)-(j) Seed coat pigmentation of the wild type, $\operatorname{ttg} 1-1, \operatorname{ttg} 1-1$ mutant expressing 35S::ArtWD40::GFP (homozygous) and ttg1-1 mutant expressing 35S::TTG1::GFP (homozygous). 
$\mathrm{CPC}$

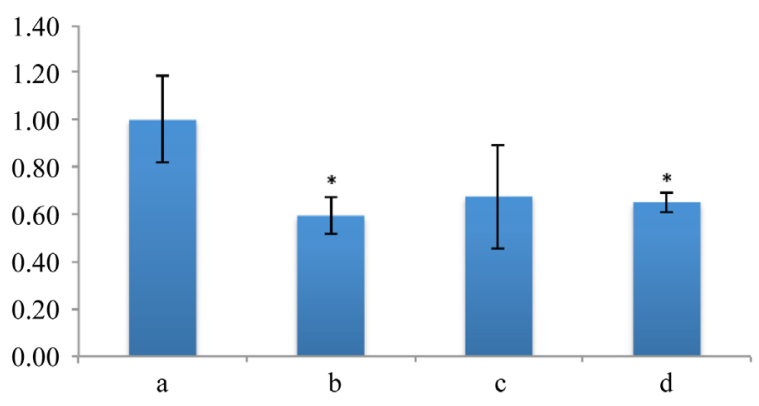

TTG2

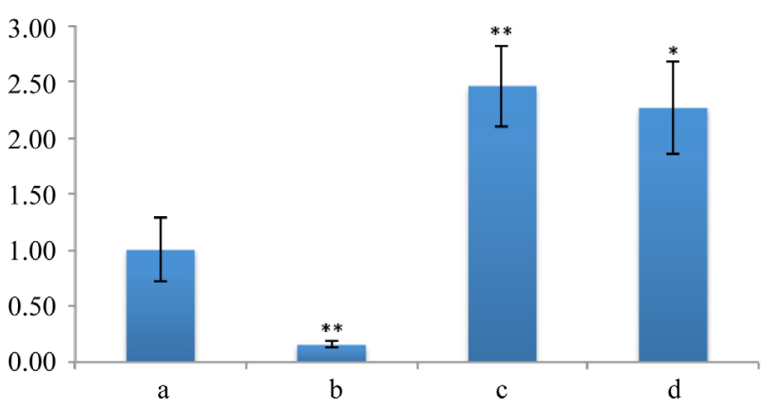

WUS

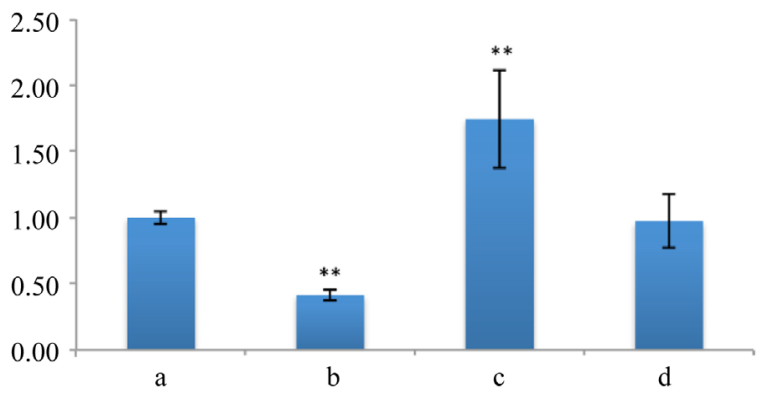

CLV2

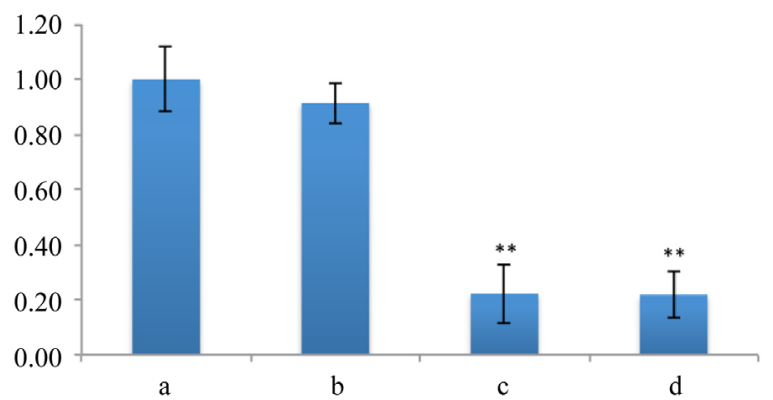

ETC1

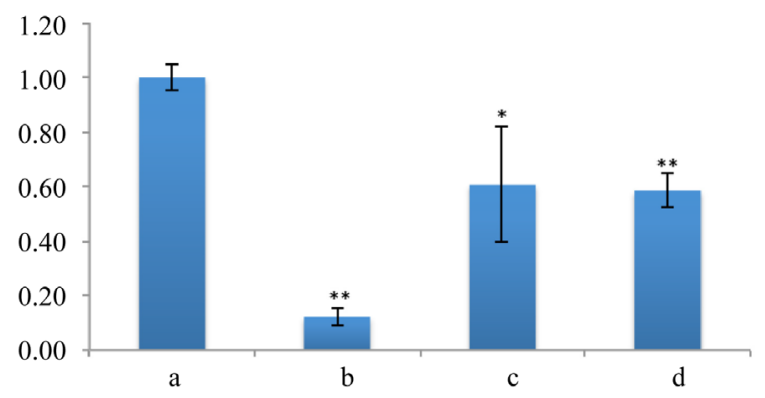

BAN

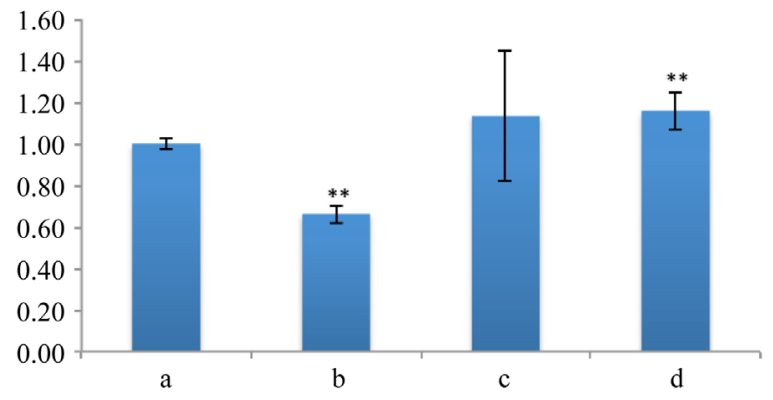

CLV1

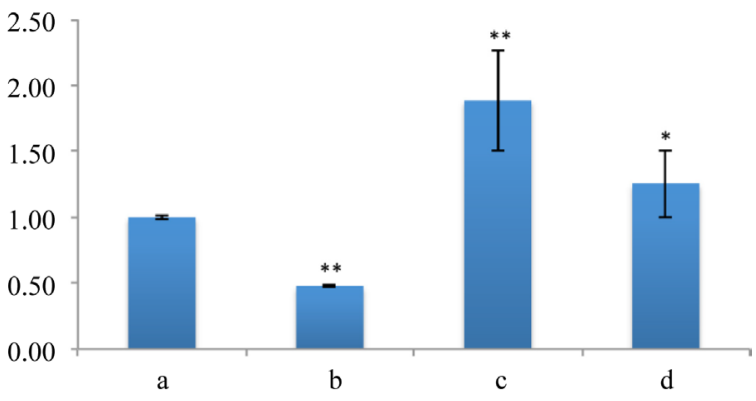

CLV3

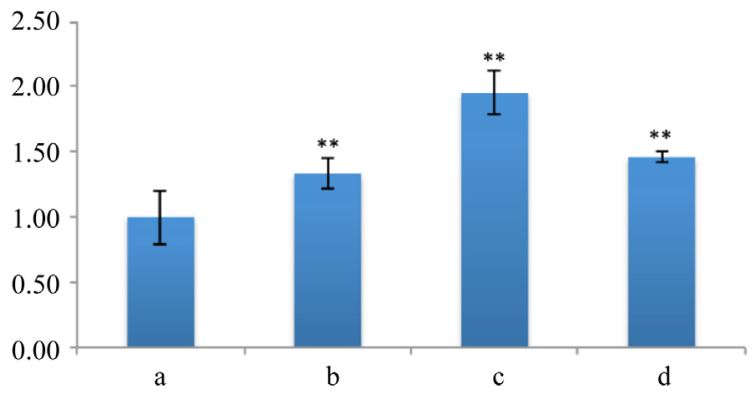

Figure 4. Genes involved in trichome formation, anthocyanidin biosynthesis and shoot meristem development were verified by qRTPCR analysis. Statistical analysis was conducted using student t-test. ** indicates significance.

\section{Materials and Methods}

\subsection{Plant Materials and Growth Condition}

Arabidopsis seeds are purchased from the Arabidopsis Biotechnology Resource Centre (Ohio State University). 
All seeds used in this study were newly harvested (within 3 months) and stored in airtight tubes after drying at $4^{\circ} \mathrm{C}$.Seeds were sterilized and germinated on MS Basal Medium (SigmaM5519) with 1\% sucrose and 0.8\% Agar.

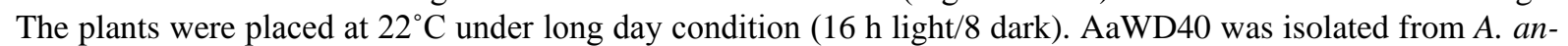
nua by PCR (forward primer, 5'-AAAGCTTAACCGAGAATGTCTCCCGACTTCTAT-3'; reverse primer, 5'-AGTCGACTCAAACTCTAAGGAGCT GCATTT G-3'). Seedlings were harvested for real-time PCR analysis using SYBR green probeat 7 day following germination unless otherwise stated. The primers used are: Imaging of YFP fusions was performed on a FluoView ${ }^{\mathrm{TM}}$ FV1000 confocal laser scanning biological microscope laser scanning microscope with excitation (488 or $514 \mathrm{~nm}$ ) and emissions (530 - $600 \mathrm{~nm}$ for GFP and 675 - 800 for RFP). Collected images were processed for maximum intensity projection. Arabidopsis cell suspension cultures (ecotype Landsbergerecta) PSB-L were grown in $250 \mathrm{ml}$ flasks at $22^{\circ} \mathrm{C}$ in a $16 \mathrm{~h} \mathrm{light} / 8 \mathrm{~h}$ dark cycle and shaked at 130 r.p.m for 3 days. Cells were sub-cultured in MS medium supplemented with $4.3 \mathrm{~g} / \mathrm{L}$ Murashige and Skoog Basal Salt Mixture, $100 \mathrm{mg} / \mathrm{liter}$ myo-inositol, $0.4 \mathrm{mg} / \mathrm{liter}$ (L?) thiamine hydrochloride, $50 \mathrm{mg} / \mathrm{liter}$ kinetin, $800 \mathrm{mg} /$ liter 1-naphthaleneacetic acid and $30 \mathrm{~g} /($ adjust $\mathrm{pH}$ to 5.7 with $\mathrm{KOH}$ ). Sub-culture was conducted by transferring $5 \mathrm{ml}$ of old cells into $45 \mathrm{ml}$ of fresh Arabidopsis MS medium every 5 days. The cultures obtained on the 3rd day after sub-culture was used for protoplast preparation.

\subsection{Transient Expression in Arabidopsis Protoplast}

Transient expression of fluorescent fusion proteins in protoplasts of suspension cell cultures were performed as described by Miao etc. [14]. For each sample, 40 ug PBI221 plasmid were mixed with protoplasts prepared from Arabidopsis thaliana (ecotype Landsbergerecta) PSB-L cell suspension cultures and used for electroporation. After electroporation, the transfected protoplasts were incubated at $27^{\circ} \mathrm{C}$ before observation under Olympus FluoView ${ }^{\mathrm{TM}}$ FV1000 confocal laser scanning biological microscope. Protoplasts were observed for fluorescent signals 8 - $12 \mathrm{~h}$ after electroporation.

\subsection{Confocal Microscopy}

Imaging of YFP fusions was performed on Olympus FluoView ${ }^{\mathrm{TM}}$ FV1000 confocal laser scanning biological microscope. Laser scanning microscope with excitation at 488 or $561 \mathrm{~nm}$ and emissions at $500-540 \mathrm{~nm}$ for GFP and 580 - 630 for RFP. Collected images were processed for maximum intensity projection.

\section{Discussion}

In this study, we conducted functional analysis of a WD40 repeat protein (AaWD40) in Arabidopsis. Interestingly, in root tip cells of transgenic Arabidopsis plants over expressing 35S::AaWD40::GFP, strong fluorescent signals were detected in both nuclear and cytoplasmic membrane. Coexpression of AtTTG1 and AaWD40 resulted in an obvious nuclear pattern. This suggests that the protein-protein interactions between WD40-repeat protein and bHLH protein may facilitate their translocation into nucleus. The fact that ectopically over expressed AaWD40 can rescue the trichomeless phenotypes of Arabidopsis mutant indicates that besides the critical roles in regulation of trichome formation and anthocyanin biosynthesis, the AaWD40 is likely involved in multiple cellular functions in Artemisia annua.

\section{Acknowledgements}

This work was supported by grants from Hong Kong UGC Research Grant Council the Hong Kong Special Administrative Region, China (Project no. CUHK 465110).

\section{References}

[1] Smith, T., Gaitatzes, C., Saxena, K. and Neer, E. (1999) The WD Repeat: A Common Architecture for Diverse Functions. Trends in Biochemical Sciences, 24, 181-185. http://dx.doi.org/10.1016/S0968-0004(99)01384-5

[2] van Nocker, S. and Ludwig, P. (2003) The WD-Repeat Protein Superfamily in Arabidopsis: Conservation and Divergence in Structure and Function. BMC Genomics, 4, 50. http://dx.doi.org/10.1186/1471-2164-4-50

[3] Galway, M., Masucci, J., Lloyd, A., Walbot, V., Davis, R. and Schiefelbein, J. (1994) The TTG Gene Is Required to Specify Epidermal Cell Fate and Cell Patterning in the Arabidopsis Root. Developmental Biology, 166, 740-754. http://dx.doi.org/10.1006/dbio.1994.1352 
[4] Gonzalez, A., Zhao, M., Leavitt, J. and Lloyd, A. (2008) Regulation of the Anthocyanin Biosynthetic Pathway by the TTG1/bHLH/Myb Transcriptional Complex in Arabidopsis Seedlings. The Plant Journal, 53, 814-827. http://dx.doi.org/10.1111/j.1365-313X.2007.03373.x

[5] Walker, A., Davison, P., Bolognesi-Winfield, A., James, C., Srinivasan, N., Blundell, T., Esch, J., Marks, M. and Gray, J. (1999) The TRANSPARENT TESTA GLABRA1 Locus, Which Regulates Trichome Differentiation and Anthocyanin Biosynthesis in Arabidopsis, Encodes a WD40 Repeat Protein. Plant Cell, 11, 1337-1350. http://dx.doi.org/10.1105/tpc.11.7.1337

[6] Zhao, M., Morohashi, K., Hatlestad, G., Grotewold, E. and Lloyd, A. (2008) The TTG1-bHLH-MYB Complex Controls Trichome Cell Fate and Patterning through Direct Targeting of Regulatory Loci. Development, 135, 1991-1999. http://dx.doi.org/10.1242/dev.016873

[7] Oppenheimer, D.G., Herman, P.L., Sivakumaran, S., Esch, J. and Marks, M.D. (1991) A Myb Gene Required for Leaf Trichome Differentiation in Arabidopsis Is Expressed in Stipules. Cell, 67, 483-493. http://dx.doi.org/10.1016/0092-8674(91)90523-2

[8] Zhang, F., Gonzalez, A., Zhao, M., Payne, C. and Lloyd, A. (2003) A Network of Redundant bHLH Proteins Functions in All TTG1-Dependent Pathways of Arabidopsis. Development, 130, 4859-4869. http://dx.doi.org/10.1242/dev.00681

[9] Buer, C. and Djordjevic, M. (2009) Architectural Phenotypes in the Transparent Test a Mutants of Arabidopsis Thaliana. Journal of Experimental Botany, 60, 751-763. http://dx.doi.org/10.1093/jxb/ern323

[10] Peer, W. and Murphy, A. (2007) Flavonoids and Auxin Transport: Modulators or Regulators? Trends in Plant Sciences, 12, 556-563. http://dx.doi.org/10.1016/j.tplants.2007.10.003

[11] Wang, W., Wang, Y., Zhang, Q., Qi, Y. and Guo, D. (2009b) Global Characterization of Artemisia Annua Glandular Trichome Transcriptome Using 454 Pyrosequencing. BMC Genomics, 10, 465. http://dx.doi.org/10.1186/1471-2164-10-465

[12] Sompornpailin, K., Makita, Y., Yamazaki, M. and Saito, K. (2002) A WD-Repeat-Containing Putative Regulatory Protein in Anthocyanin Biosynthesis in Perilla frutescens. Plant Molecular Biology, 50, 485-495. http://dx.doi.org/10.1023/A:1019850921627

[13] Baudry, A., Heim, M.A., Dubreucq, B., Caboche, M., Weisshaar, B. and Lepiniec, L. (2004) TT2, TT8, and TTG1 Synergistically Specify the Expression of BANYULS and Proanthocyanidin Biosynthesis in Arabidopsis Thaliana. Plant Journal, 39, 366-380. http://dx.doi.org/10.1111/j.1365-313X.2004.02138.X

[14] Miao, Y. and Jiang, L. (2007) Transient Expression of Fluorescent Fusion Proteins in Protoplasts of Suspension Cultured Cells. Nature Protocols, 2, 2348-2353. http://dx.doi.org/10.1038/nprot.2007.360 